\title{
STUDIES ON MARINE MICROORGANISMS. I
}

\author{
ISOLATION FROM THE JAPAN SEA
}

\author{
Yoshiro OKami and TaKao OKazaKi \\ Institute of Microbial Chemistry, Shinagawa-ku, Tokyo, Japan
}

(Received for publication May 8, 1972)

In this study, 58 samples of water and mud were collected at 19 different locations in the Japan Sea, and from these 142 strains of microorganisms were isolated. In all 126 isolates were bacteria and 16 isolates were yeast and fungi. No actinomycetes were isolated. The isolates were placed into 10 groups in accordance with SHEwAN's scheme. A total of 35 strains $(28.5 \%)$ of 123 isolates were dependent to sea water, and 25 of these strains $(20.3 \%)$ showed antimicrobial activities.

Few examples of antibiotic activities from marine microorganisms have been reported ${ }^{4,5,6)}$, while various kinds of antibiotics have been obtained mainly from different terrestrial microorganisms. Since environmental conditions of the sea are extremely different from terrestrial conditions, it is supposed that microorganisms in the sea have different characteristics from known terrestrial microorganisms and hence, might produce different types of antibiotics from those we know. Microorganisms were isolated from samples collected in the Japan Sea and placed by their characteristics into 10 groups. Their antibiotic activity was examined under various conditions.

\section{Materials and Methods}

In all, 58 samples were collected with the help of the Science and Technology Agency and the Maritime Safety Agency of Japan. Sea water and mud were collected at 19 different locations (Table 1, Fig. 1) in the Japan Sea during the cruise of coast guard vessel "Takuyo-maru" in August, 1969.

Sampling Methods: Water samples were collected with the aid of NANSEN's sampler and mud samples with a core sampler. These samples were aseptically stored in $250 \mathrm{ml}$ polyethylene bottles and kept in a cold box $\left(5^{\circ} \mathrm{C}\right)$ during the cruise.

Methods of Isolating Microorganisms : A $100-\mathrm{ml}$ aliquot of each water sample was centrifuged at $1,000 \mathrm{rpm}$ for 10 minutes

Fig. 1. Locations in the Japan Sea for the collection of sea water and mud.

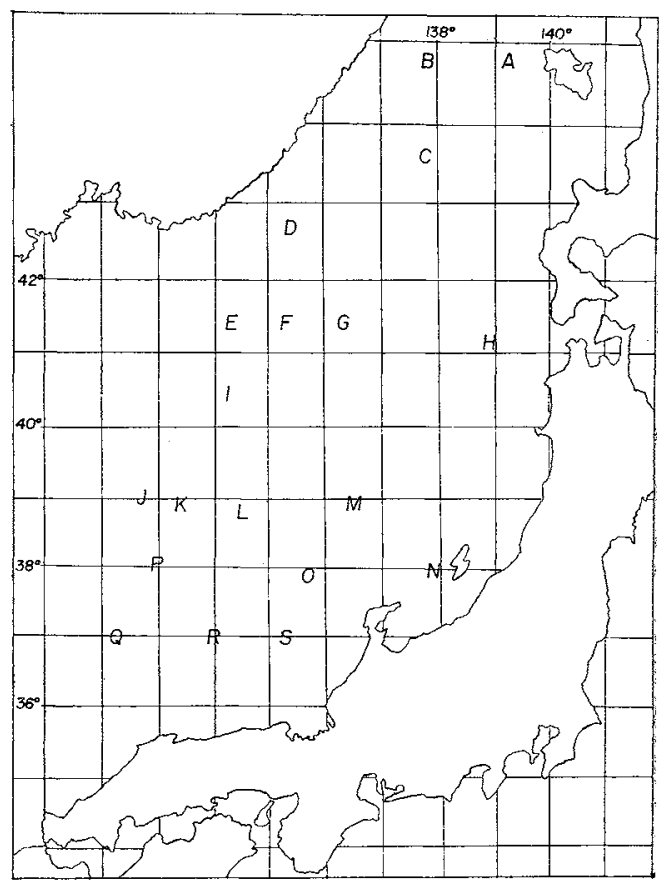


and aseptically filtered with a Millipore membrane filter (pore size $0.3 \mu$, $\mathrm{PH}$ type). Concentrated microorganisms on the filter were suspended in $2.5 \mathrm{ml}$ of the filtered sea water. A $0.5-\mathrm{ml}$ aliquot of the suspension was spread over four kinds of agar media of different composition (Fig. 2). These media were cultured for one to two weeks at $22^{\circ} \mathrm{C}$, and the colonies which appeared were selected and transplanted to an agar slant of the same composition as above. Sea mud samples were spread directly over the agar media.

\begin{tabular}{cll} 
Medium & \multicolumn{2}{c}{ Components (\%) } \\
$\mathrm{K}$ & Glucose & 1.0 \\
& Asparagine & 0.05 \\
& $\mathrm{~K}_{2} \mathrm{HPO}_{4}$ & 0.05 \\
& Agar & 1.7 \\
$\mathrm{~N}$ & HH 7.4 & \\
MYS & Maltose & 1.0 \\
& Yeast extract & 0.4 \\
& Agar & 1.7 \\
ZD & pH 7.2 & \\
& Bacto-peptone & 0.5 \\
& Ferric phosphate & 0.01 \\
& Bacto-yeast extract & 0.1 \\
& Agar & 1.7 \\
& pH 7.6 &
\end{tabular}

Z Same as ZD (artificially mixed sea water* instead of distilled water in ZD)

* Jamarine Co., Ltd., Osaka

Antimicrobial Activity: Isolates were inoculated into the following three media and shaken at $22^{\circ} \mathrm{C}$ for 4 days. The broths were examined for antimicrobial activity by the cylinder plate method against following test microorganisms, Staphylococcus aureus 193, Escherichia coli NIHJ, Mycobacterium 607, and Candida albicans and Phytophthora capsici.

\begin{tabular}{cll} 
Medium & \multicolumn{2}{c}{ Components $(\%)$} \\
$\mathrm{V}$ & Glucose & 0.5 \\
& Peptone & 1.0 \\
& Yeast extract & 0.5 \\
& $\mathrm{~K}_{2} \mathrm{HPO}_{4}$ & 0.05 \\
$\mathrm{MgSO}_{4}$ & 0.05 \\
& $\mathrm{NaCl}$ & 2.0 \\
$\mathrm{pH} 7.8$ &
\end{tabular}

Table 1. Location in the Japan Sea for the collection of sea water and mud with reference to group of the isolated microorganisms.

\begin{tabular}{|c|c|c|c|c|c|}
\hline $\begin{array}{l}\text { Sta- } \\
\text { tion }\end{array}$ & Date & $\begin{array}{c}\text { North } \\
\text { latitude }\end{array}$ & $\begin{array}{l}\text { East } \\
\text { longi- } \\
\text { tude }\end{array}$ & $\begin{array}{c}\text { Depth } \\
(\mathrm{m})\end{array}$ & Group** \\
\hline A & 44.7. 1 & $44^{\circ} 45^{\prime}$ & $139^{\circ} 20^{\prime}$ & $\begin{array}{c}\text { Surface } \\
400 \\
1,000 \\
1,520 *\end{array}$ & $\begin{array}{l}\mathrm{F}_{1} \\
\text { Yeast, Fungi } \\
\text { Fungi } \\
\mathrm{B}, \mathrm{D}, \text { Fungi }\end{array}$ \\
\hline B & 7. 2 & $44^{\circ} 45^{\prime}$ & $137^{\circ} 52^{\prime}$ & $\begin{array}{c}\text { Surface } \\
400 \\
1,000\end{array}$ & - \\
\hline $\mathrm{C}$ & 7. 3 & $43^{\circ} 27^{\prime}$ & $137^{\circ} 44^{\prime}$ & $\begin{array}{c}\text { Surface } \\
400 \\
1,000\end{array}$ & $\begin{array}{l}\overline{F_{1}} \\
\mathrm{~F}_{2}\end{array}$ \\
\hline $\mathrm{D}$ & 7. 5 & $42^{\circ} 29^{\prime}$ & $135^{\circ} 23^{\prime}$ & \begin{tabular}{|c|} 
Surface \\
400 \\
1,000
\end{tabular} & $\begin{array}{l}\mathrm{F}_{1} \\
\mathrm{~B} \\
\mathrm{~F}_{4}\end{array}$ \\
\hline $\mathrm{E}$ & 7. 6 & $41^{\circ} 16^{\prime}$ & $134^{\circ} 16^{\prime}$ & $\begin{array}{c}\text { Surface } \\
400 \\
1,000\end{array}$ & $\begin{array}{l}F_{1}, F_{2} \\
\text { Fungi } \\
A, F_{1}\end{array}$ \\
\hline$F$ & 7. 6 & $41^{\circ} 16^{\prime}$ & $135^{\circ} 11^{\prime}$ & \begin{tabular}{|c|} 
Surface \\
400 \\
1,000
\end{tabular} & $\begin{array}{l}\mathrm{B}, \text { Fungi } \\
\mathrm{F}_{1}, \mathrm{~F}_{2} \\
\mathrm{~B}, \text { Fungi }\end{array}$ \\
\hline G & 7. 7 & $41^{\circ} 14^{\prime}$ & $136^{\circ} 18^{\prime}$ & $\begin{array}{l}3,300 \\
3,720 *\end{array}$ & $\bar{B}$ \\
\hline $\mathrm{H}$ & 7. 8 & $41^{\circ} 01^{\prime}$ & $138^{\circ} 55^{\prime}$ & $\begin{array}{c}\text { Surface } \\
400 \\
1,000\end{array}$ & $\begin{array}{l}\mathrm{F}_{8}, \text { Fungi } \\
\mathrm{D}\end{array}$ \\
\hline I & 7.14 & $40^{\circ} 16^{\prime}$ & $134^{\circ} 13^{\prime}$ & \begin{tabular}{|c|} 
Surface \\
400 \\
1,000
\end{tabular} & $\begin{array}{l}A, B, D, F_{4} \\
A, B \\
F_{1}, F_{2}\end{array}$ \\
\hline $\mathrm{J}$ & 7.15 & $38^{\circ} 53^{\prime}$ & $132^{\circ} 45^{\prime}$ & $\begin{array}{c}\text { Surface } \\
400 \\
1,000\end{array}$ & $\begin{array}{l}\text { Fungi } \\
\mathrm{F}_{4} \\
\mathrm{~A}, \mathrm{D}\end{array}$ \\
\hline $\mathrm{K}$ & 7.15 & $38^{\circ} 44^{\prime}$ & $133^{\circ} 22^{\prime}$ & $\begin{array}{c}\text { Surface } \\
400 \\
1,000\end{array}$ & $\begin{array}{l}\mathrm{A} \\
\text { Fungi } \\
\mathrm{F}_{3}\end{array}$ \\
\hline $\mathrm{L}$ & 7.16 & $38^{\circ} 37^{\prime}$ & $134^{\prime} 30^{\prime}$ & $2,950 *$ & $\mathrm{C}, \mathrm{D}, \mathrm{F}_{3}$ \\
\hline$M$ & 7.16 & $38^{\circ} 51^{\prime}$ & $136^{\circ} 33^{\prime}$ & $\begin{array}{c}\text { Surface } \\
400 \\
1,000 \\
2,630^{*}\end{array}$ & $\begin{array}{l}\overline{\mathrm{D}} \\
\mathrm{A}, \mathrm{F}_{4} \\
\mathrm{~B}, \mathrm{~F}_{1}, \mathrm{~F}_{2}\end{array}$ \\
\hline $\mathrm{N}$ & 7.17 & $37^{\circ} 45^{\prime}$ & $137^{\circ} 52^{\prime}$ & $\begin{array}{c}\text { Surface } \\
400 \\
1,000 \\
1,640\end{array}$ & $\begin{array}{l}\mathrm{D} \text {, Fungi, Yeast } \\
\mathrm{A} \text {, Yeast } \\
\mathrm{F}_{4}, \mathrm{~B}, \mathrm{C}, \mathrm{D}, \mathrm{F}_{4} \\
\mathrm{~A}, \mathrm{~B}\end{array}$ \\
\hline 0 & 7.19 & $37^{\circ} 43^{\prime}$ & $135^{\circ} 42^{\prime}$ & $\begin{array}{c}\text { Surface } \\
400 \\
1,000 \\
2,830^{*}\end{array}$ & $\begin{array}{l}F_{1}, F_{2}, \text { Fungi } \\
\bar{A} \\
A, \text { Fungi }\end{array}$ \\
\hline$P$ & 7,19 & $37^{\circ} 54^{\prime}$ & $133^{\circ} 02^{\prime}$ & $\begin{array}{c}\text { Surface } \\
400\end{array}$ & $\begin{array}{l}A, B \\
F_{3}, \text { Fungi }\end{array}$ \\
\hline $\mathrm{Q}$ & 7.21 & $36^{\circ} 49^{\prime}$ & $132^{\circ} 16^{\prime}$ & $\begin{array}{c}\text { Surface } \\
400 \\
1,000 \\
1,540^{*}\end{array}$ & $\begin{array}{l}\mathrm{A}, \mathrm{B}, \mathrm{D}, \mathrm{F}_{2} \\
\mathrm{~A}, \mathrm{~B}, \mathrm{~F}_{3} \\
\mathrm{~A}, \mathrm{~B}, \mathrm{C}, \mathrm{F}_{1} \\
\mathrm{C}, \mathrm{F}_{1}, \mathrm{~F}_{4}\end{array}$ \\
\hline $\mathrm{R}$ & 7.22 & $36^{\circ} 53^{\prime}$ & $134^{\circ} 02^{\prime}$ & $\begin{array}{c}\text { Surface } \\
400 \\
1,000 \\
1.720\end{array}$ & $\begin{array}{l}\text { Yeast } \\
\mathrm{F}_{3} \\
\mathrm{~F}_{4} \\
\mathrm{~A}, \mathrm{C}, \mathrm{F}_{2}\end{array}$ \\
\hline $\mathrm{S}$ & 7.22 & $36^{\circ} 53^{\prime}$ & $135^{\circ} 16^{\prime}$ & $\begin{array}{c}\text { Surface } \\
400 \\
1,000\end{array}$ & $\begin{array}{l}\mathrm{B}, \mathrm{F}_{4} \\
\mathrm{~F}_{1} \\
\mathrm{~B}\end{array}$ \\
\hline
\end{tabular}


Fig. 2. Isolation of marine microorganisms from sea water.

Sample, $100 \mathrm{ml}$ (Sea water)

Centrifuge, $1,000 \mathrm{rpm} .10 \mathrm{~min}$.

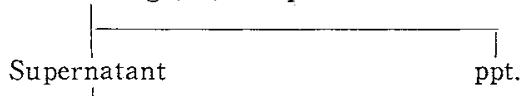

$50 \mathrm{ml}$

Membrane filter, $0.3 \mu$

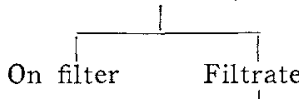

$+$

$2.5 \mathrm{ml}$

1

spread

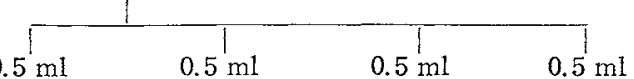

$\mathrm{K}$ medium $\mathrm{MYS}$ medium $\mathrm{ZD}$ medium $\mathrm{Z}$ medium
Table 2. Classification of isolates from the Japan Sea

\begin{tabular}{c|l|c}
\hline Group & \multicolumn{1}{|c|}{ Main genera } & $\begin{array}{c}\text { Number of } \\
\text { isolates }\end{array}$ \\
\hline $\mathrm{A}$ & $\begin{array}{l}\text { Paracolobacterium } \\
\text { Escherichia } \\
\text { Achromobacter } \\
\text { Alcaligenes }\end{array}$ & 22 \\
$\mathrm{C}$ & $\begin{array}{l}\text { Flavobacterium, Cytophaga } \\
\mathrm{D}\end{array}$ & 18 \\
$\mathrm{E}$ & Vibrio & 14 \\
$\mathrm{~F}_{1}$ & Aeromonas & 12 \\
$\mathrm{~F}_{2}$ & Pseudomonas type 1 & 0 \\
$\mathrm{~F}_{3}$ & Pseudomonas type 2 & 15 \\
$\mathrm{~F}_{4}$ & Pseudomonas type 3 & 6 \\
$\mathrm{G}$ & Strains not grouped in & 14 \\
& SHEwan's scheme & 19 \\
Yeast & & 5 \\
Fungi & & 11 \\
\hline Total & & 142 \\
\hline
\end{tabular}

Table 3. Growth dependence to sea water

\begin{tabular}{c|c|c}
\hline Group & Tested & Dependent* \\
\hline $\mathrm{A}$ & 22 & 4 \\
$\mathrm{~B}$ & 18 & 5 \\
$\mathrm{C}$ & 13 & 3 \\
$\mathrm{D}$ & 11 & 2 \\
$\mathrm{E}$ & 0 & 0 \\
$\mathrm{~F}_{1}$ & 15 & 4 \\
$\mathrm{~F}_{2}$ & 6 & 2 \\
$\mathrm{~F}_{3}$ & 6 & 4 \\
$\mathrm{~F}_{4}$ & 14 & 7 \\
$\mathrm{G}$ & 13 & 4 \\
Yeast & 5 & 0 \\
Fungi & 0 & 0 \\
\hline Total & 123 & 35 \\
\hline
\end{tabular}

* No growth in nutrient broth (Distilled water is used instead of sea water).

$\begin{array}{ll}\text { Xlucose } & 0.5 \\ \text { Soy bean meal } & 1.5 \\ \mathrm{~K}_{2} \mathrm{HPO}_{4} & 0.05 \\ \mathrm{MgSO}_{4} & 0.05 \\ \mathrm{NaCl} & 2.0 \\ \mathrm{pH} 7.8 & \end{array}$

Table 4. Antimicrobial activity of marine microorganisms

\begin{tabular}{c|c|c|c|c|c|c}
\hline & \multirow{2}{*}{ Group } & Tested & Active* & \multicolumn{3}{|c|}{ Active against microorganisms** } \\
\cline { 4 - 7 } & & & Staph. & E. coli & Mycob. & Fungi \\
\hline A & 22 & 4 & & & & 4 \\
B & 18 & 3 & & & 1 & 2 \\
C & 13 & 1 & & & & 1 \\
D & 11 & 2 & & 1 & & 1 \\
$F_{1}$ & 15 & 5 & & 1 & 1 & 3 \\
$F_{2}$ & 6 & 2 & & 1 & & 1 \\
$F_{3}$ & 6 & 2 & & & & 2 \\
$F_{4}$ & 14 & 5 & & 1 & 3 & 4 \\
G & 13 & 0 & & & & 1 \\
Yeast & 5 & 1 & & 1 & & 19 \\
\hline Total & 123 & 25 & 0 & 5 & 5 & 19 \\
\hline
\end{tabular}

* Active to either bacteria or fungi tested.

** Staph.: Staphylococcus aureus 193

E. coli: Escherichia coli NIHJ.

Mycob. : Mycobacterium 607.

Fungi : Candida albicans and Phytophthora capsici.
Z Bacto-peptone $\quad 0.5$

Bacto-yeast exract $\quad 0.01$

Ferric phosphate 0.1

Artificially mixed sea water

$750 \mathrm{ml}$ and dist. water $250 \mathrm{ml}$ $\mathrm{pH} 7.8$

\section{Results and Discussion}

Grouping of Isolated Marine Microorganisms

On the basis of morphological and physiological characteristics, 142 isolated strains were placed into 10 groups namely, from A to $G$ according to SHewan's scheme $^{1)}$ (Fig. 3). Each of the groups contains the following genera; A (Paracolobacterium, Escherichia); B (Achromobacter, Alcaligenes); C (Flavobacterium, Cytophaga); 
Fig. 3. Classification scheme of marine bacteria

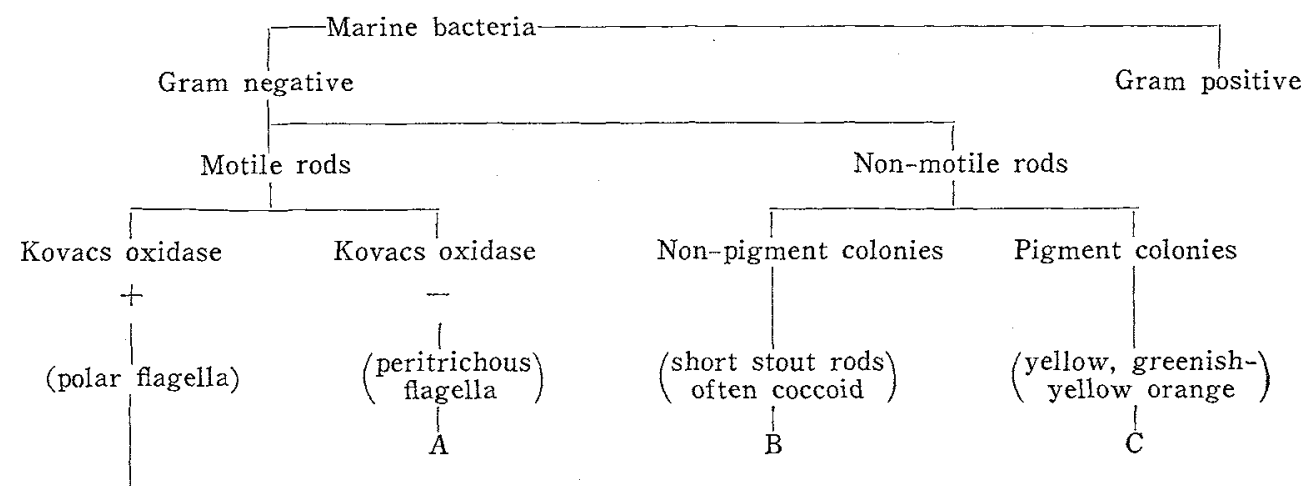

HugH and LeIrsons test

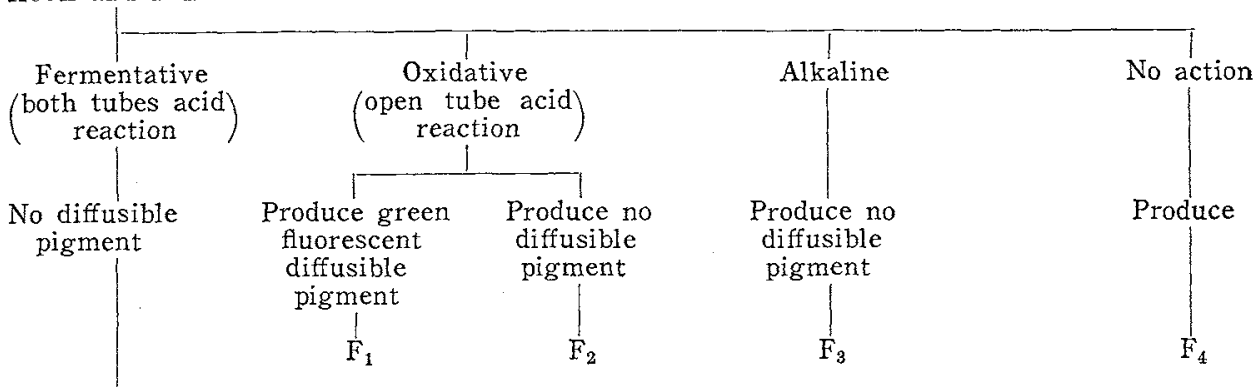

Acid and no gas

in glucose

$\left(\begin{array}{c}\text { some strains } \\ \text { produce small }\end{array}\right)$

trace of gas

Sensitive to

the pteridine

compound

(0/129)

D
Acid and large amounts of gas in glucose at $20^{\circ} \mathrm{C}$

Insensitive to pteridine compound $(0 / 129)$

E
$\mathrm{D}$ (Vibrio); E (Aeromonas); $\mathrm{F}_{1}$ (Pseudomonas type 1 ); $\mathrm{F}_{2}$ (Pseudomonas type 2 ); $\mathrm{F}_{3}$ (Pseudomonas type 3 ); $F_{4}$ (Pseudomonas type 4); G (Microorganisms not grouped by SHEw AN's scheme); yeast and fungi (Table 2).

The results suggest the regional difference of microorganisms in the Japan Sea as

shown in Table 1. Among the isolates, bacteria, especially Gram-negative bacteria, were seen more often than other microorganisms such as fungi and yeast. No actinomycetes were isolated from the Japan Sea in this experiment, while many actinomycetes have been isolated by other investigators from the North Atlantic Ocean and the Pacific Ocean ${ }^{2,3,4}$. Failure to isolate actinomycetes would be partly dependent on the period preserving samples at $5{ }^{\circ} \mathrm{C}$ more than one year and partly on the scarcity of actinomycetes in the deep sea.

Growth Dependence on Sea Water

In all, 123 strains of isolated marine microorganisms were inoculated onto two media, Bacto-nutrient broth (Bacto-beef extract $0.3 \%$, Bacto-peptone 0.5\%, pH 6.8) and sea water Bacto-nutrient broth (prepared artificially with sea water instead of distilled water as above) and cultured for 1 week at $22^{\circ} \mathrm{C}$. As shown in Table 3, 35 strains $(28.5 \%)$ were dependent on sea water.

\section{Antimicrobial Activity of Marine Microorganisms}

There have been few reports on the antimicrobial activity of marine microorganisms ${ }^{4,5,6)}$. Here, 123 strains of marine microorganisms were examined for possible 
antimicrobial activity by using three kinds of medium. The results are shown in Table 4. It is of interest to note that these marine microorganisms exhibit poor activity against Gram-positive bacteria in comparison with the terrestrial microorganisms ever isolated. The fact that more than 20 percent of marine microorganisms tested showed antimicrobial activities, suggests that they would be worthy to study as sources for biologically active principles.

\section{Acknowledgements}

The authors wish to thank deeply the Science and Technology Agency and the Maritime Safety Agency of Japan for their help in collecting samples. They also wish to thank Dr. H. UmezAwA for his valuable advice and encouragement, and Miss H. Namamura and Miss H. Shimazu for their technical assistance.

\section{References}

1) Shewan, J. M.: The differentiation of certain genera of Gram-negative bacteria frequently encountered in marine environment. In C. H. OpPENHEIMER (ed.), Marine microbiology. Charles C. Thomas, Publisher, Springfield. pp. 499 521, 1963

2) Weyland, H.: Actinomycetes in North Sea and Atlantic Ocean sediments. Nature $223: 858$, 1969

3) Wexiand, H. : Studies on Actinomycetes of the sea benthos. Presented at the Ocean WorldJoint Oceanographic Assembly, Tokyo, 1970

4) Grein, A. \& S. P. Meyers : Growth characteristic and antibiotic production of Actinomycetes isolated from littoral sediments. J. Bact. $76: 457 \sim 463,1958$

5) Rosenfeld, W. D. \& C. E. Zobeli: Antibiotic production by marine microorganisms. J. Bact. $54: 393 \sim 398,1947$

6) Burkholder, R. \& G. M. Sharma: Antimicrobial agents from the sea. Lloydia $32: 466 \sim 483$, 1969 